\title{
Posicionamiento del valor de marca de firmas de moda
}

Brand Equity

Positioning Of

Fashion Firms

Estela Núñez Barriopedro

Universidad de Alcalá

estela.nunezb@uah.es

Pedro Cuesta Valiño

Universidad de Alcalá

pedro.cuesta@uah.es

Pablo Gutiérrez Rodríguez

Universidad de León

Pablo.gutierrez@unileon
Núñez, E., Cuesta, P. y Gutiérrez, P. (2012)

Posicionamiento del valor de marca de firmas de moda. Revista Internacional de Investigación en Comunicación aDResearch ESIC. № 7 Vol 7. Primer semestre, enero-junio 2013 · Págs. 8 a 19 DOI: 10.7263/ADR.RSC.007.01 


\section{RESUMEN}

Clasificación JEL:

M310

\section{Palabras clave:}

Posicionamiento, gestión de marca, comunicación, sector textil, firmas de moda
En la actualidad la marca es un instrumento clave de valor añadido en las empresas. Una marca fuerte es un instrumento que permite crear y mantener una valiosa ventaja competitiva en entornos competitivos y globalizados. Esta investigación analiza el valor de las marcas desde la perspectiva del detallista con una metodología objetiva que permite posicionar una marca respecto a sus principales competidoras y permite descubrir cuáles son las principales estrategias de diferenciación de cada una de las firmas. Este estudio se ha aplicado en el sector textil aunque podría extenderse a otros sectores de actividad.

\section{ABSTRACT}

\section{JEL Classification:}

\section{M310}

\section{Key words:}

Positioning,

brand management, communication, textile sector, fashion firms
Currently, the brand is a key value-adding tool for companies. A strong brand is a tool that allows one to create and maintain a valuable competitive advantage in competitive and globalized spheres. This research analyses the value of brands from the retailer's perspective using an objective methodology that allows brand positioning against its main competitors and allows us to discover what the main differentiating strategies of each of the firms are. This study has been applied to the textile sector although it could also be used for other business sectors. 


\section{Introducción}

En el entorno actual nos encontramos cada vez con mercados más competitivos y globalizados. Por lo que las organizaciones se plantean el reto de diferenciarse o morir. Ante esta situación la marca se convierte en instrumento clave como estrategia de comunicación del valor añadido de las empresas. Una marca fuerte es la forma más eficiente de crear y mantener una valiosa ventaja competitiva.

Además una marca fuerte puede provocar grandes diferencias en la elección del consumidor de un producto sobre otros aparentemente iguales. Esta investigación analiza el valor añadido de las marcas desde la perspectiva del detallista. El valor de la marca también puede ser valorado desde la óptica del consumidor.

El presente trabajo se centra en el sector de la moda porque es un sector con buenas expectativas de crecimiento y recuperación. Concretamente se espera que haya un crecimiento de hasta el 5,4\% en el sector textil y de la confección en el período 2007-2012, según Datamonitor. El impulso de la demanda exterior ha sido fundamental para la progresiva mejora de la situación coyuntural del sector textil y de la confección, superando así el punto amargo de la crisis, lo que se refleja en una mejor evolución de la actividad y el empleo durante la primera parte del año 2010, siempre en comparación con los datos muy negativos de 2009 en los que la producción se vio seriamente dañada; la recuperación, según Mintel, es lenta. El sector de los productos textiles y de la confección representa una industria muy importante y bien establecida que, además, ejerce un impulso dinamizador de la economía española.

\section{Marco teórico}

El valor de marca es un concepto que ha sufrido una evolución importante en su entendimiento y en las variables que la componen.

Existe una gran variedad de enfoques sobre métodos de valoración de marca. Muchos son los autores que han dado su propio punto de vista sobre la medición de valor de marcas. A continuación se hace una revisión de los mismos, siendo conscientes de las limitaciones de cada uno de ellos:

Para Aaker(1991, 1996) el valor de marca se mide en cinco categorías: fidelidad, reconocimiento del nombre, calidad percibida, asociaciones adicionales a la calidad percibida y otros activos relacionados con el comportamiento del mercado.

La Valoración por el coste actual o de reposición, se define coste actual como el equivalente a lo que un tercero está dispuesto a pagar por una marca, o al coste del proceso de consolidación de ésta partiendo de cero (Arnold ,1994). Para la utilización de este método es importante considerar parámetros como la imagen, notoriedad, cuota de mercado, liderazgo, etc. y se establecen dos hipótesis: la primera sobre el coste de lanzar al mercado una marca con éxito similar a la que estemos valorando, y la segunda relativa a las probabilidades de alcanzar dicho éxito al coste previsto (Yagüe, M.J. 1996).

En el Método del valor de mercado, el valor de la marca se establece a partir de los valores de marcas similares en el mercado (Srivastava, 1990 y Murphy, 1991). Una vía para calcular el valor de la marca en éste método sería a través de la investigación a clientes, interrogándolos acerca de cuánto estarían dispuestos a pagar por ciertos atributos o características de un producto o ser- 
vicio (una de éstas características sería el nombre de la marca).

La Valoración por los potenciales beneficios futuros, se centra en la rentabilidad futura materializada en los cash flows futuros. En este caso el valor de marca se corresponde con el valor actual de los beneficios futuros. El principal inconveniente de este método es que al proyectarse el flujo de caja no se tiene en cuenta los posibles cambios en el entorno del mercado. Es factible que el flujo de caja no dependa enteramente de la marca sino de las habilidades administrativas y de marketing de los gerentes de marca y en otros casos, del sistema de distribución diseñado para la marca (Murphy, 1991 y Aaker, 1994).

El Método Precio Primado, por medio de la aplicación de un múltiplo apropiado a las ganancias derivadas de la marca, este método cumple con los requisitos del marketing, finanzas y legales. Se toma como base el nivel actual de rentabilidad, expresado como un promedio ponderado de las utilidades generadas en los últimos años. Existen siete factores ponderados: liderazgo, estabilidad o longevidad, mercado, internacionalización, tendencia, soporte, protección. Estos factores dan pie para la configuración de una marca ideal contra la cual se comparan los resultados de la marca permitiendo establecer un valor. La marca ideal es la marca libre de riesgo para su evaluación. Este método se basa en el cálculo del precio extra de un producto con marca respecto a otro producto con características análogas sin marca, es decir, se valora el precio de venta que puede lograr una marca, por encima de los precios de la competencia (Cerviño y Núñez, 2011).

Un enfoque implementado por los profesores de finanzas Carol J., Simón y Mary Sullivan de la Universidad de Chicago, es el precio de las accio- nes que calcula el valor de la marca en función del valor financiero de mercado de la empresa; es decir, se tiene en cuenta el precio de las acciones, basándose en la idea de que el mercado bursátil ajustará el precio de la empresa reflejando las expectativas futuras de sus marcas. El valor de mercado de la compañía está en función del precio y de la cantidad de acciones. El valor de la marca se asume en función de la antigüedad y el orden de entrada al mercado de la misma, es decir, a mayor antigüedad mayor valor, la publicidad acumulada crea valor, y la participación en la industria, ya que se relaciona con ventajas de posicionamiento.

Un método propuesto por Erloz (1995) es la aproximación al valor de la marca a través del modelo de elección del consumidor. Este método permite medir el valor que los consumidores asignan a la marca. Para conseguir este objetivo, el modelo agrupa a los consumidores de un mercado en segmentos cuyo comportamiento sea homogéneo en su proceso de elección de una marca y relaciona, para cada uno de los segmentos, la utilidad proporcionada por la compra con las asociaciones de marca-producto del consumidor, con las características físicas de la marca del producto, y las acciones de marketing que se desarrollan a corto plazo para dicha marca.

Otro método para medir la causa del valor de marca es el análisis conjunto. Esta técnica permite descomponer la utilidad de un producto para el consumidor en componentes (Rangaswamy, 1993): la utilidad derivada de los atributos físicos del producto, la derivada de la presencia de marca y la resultante de la interacción entre marca y los atributos del producto. Como alternativa del análisis conjunto como método de valoración del capital de marca, autores como Park y Srinivasan (1994) y Gómez Arias (1995) propo- 
nen un método que permite estudiar simultáneamente la influencia de la marca y de sus componentes en las preferencias de los consumidores. El problema fundamental de éste método es que integra información de los atributos de forma individualizada y no a través de un modelo conjunto que contemple posibles interacciones entre ellos.

El Método de valoración de la contabilidad de momentos, desarrollado por Farquhar e Ijiri (1993), considera que una medida sobre el funcionamiento de una marca, comúnmente utilizada, son sus ventas.

El Método de valoración por el coste teórico, considera a la marca como el valor de las inversiones realizadas a lo largo del tiempo. Favorece a aquellas empresas en las que su valor de marca se ha creado a partir del marketing y de la publicidad. Este método se obtiene calculando la suma de todos los costes o inversiones relacionadas con su comunicación, distribución, investigación y desarrollo, etc., durante un periodo de tiempo determinado (Kapferer, 1992 y Arnold, 1992).

Podríamos afirmar que el valor de marca es el conjunto de percepciones y experiencias favorables o desfavorables (características, cualidades y beneficios), que generan una imagen específica en la mente del consumidor a partir de la personalidad y posicionamiento que detenta la marca, el cual añade o no valor al producto o servicio diferenciándolo como único.

En la presente investigación se trata de analizar cómo la marca crea valor para la empresa y medir esta creación en relación con la competencia.

El sector objeto de estudio es el sector textil porque es un sector muy dinámico con buenas expectativas de crecimiento y recuperación. Del
2004 al 2008, en España, se produjo un incremento del consumo de productos textiles, sin embargo, a partir del 2009 el consumo se empezó a reducir. Aunque se espera que haya un crecimiento de hasta el 5,4\% en el período 20072012, según Datamonitor.

Por lo general, el consumidor español prefiere productos textiles y de confección de marcas nacionales. Normalmente suelen asociar la calidad de los productos al dinero, siendo conscientes de la marca. Debido al fenómeno de los precios bajos compañías como H\&M, Primark, C\&A y algunas cadenas de Inditex ofrecieron diversos tipos de ropas de bajo coste. Esto se debió a la posibilidad de producir en China e India, principalmente, donde la mano de obra unida a los volúmenes de ropa es más económico, además de no poseer grandes campañas publicitarias, lo que te permitió tener un producto en tendencia, con independencia de que la prenda solo dure una temporada. Con esto las ventas crecieron debido al exhaustivo control de gastos de su red de ventas, pero las tiendas de ropa tradicionales (Mango, Cortefiel, Trucco o Benetton) vieron disminuidas las ventas por lo que se intentaron adaptar al mercado. Debido a este fenómeno competitivo los consumidores pueden disfrutar de una oferta cada vez mayor.

Este sector ha sido objeto de estudio sobre ranking de marcas. Entre los principales resultados destacan que Zara e Inditex, en el 2009, se encontraban en el ranking de las 50 marcas más valiosas del mundo. Zara, en ese momento, se encontraba por detrás de las marcas Louis Vuitton, Gucci, Nike y H\&M (en el puesto 21). Sin embargo Inditex, en el puesto 50, superó a su gran competidor Gap, que se situaba en el 78. En marzo, Zara quedó situada en el puesto número 5 del ranking Best Retail Brands de Inter- 
brand (Cerviño, 2010), en el que también se colaron las españolas El Corte Inglés y Mango.

Hay que mencionar que Zara ha ido subiendo puestos entre las marcas más valiosas del mundo, según el ranking de Interbrand. En 2010 ocupa el puesto 48, con 7.468 millones de dólares, un $10 \%$ más que en 2009 . Respecto a marcas de moda, se sitúa por delante de Gap (84) o Armani (77), y por detrás de Louis Vuitton, H\&M (21), Nike o Gucci.

Según Millward Brown Optimor, H\&M, Louis Vuitton y Wall-Mart son las marcas más valiosas del mundo en sus respectivos sectores en 2009. Para ello tuvieron en cuenta la capacidad que tienen las empresas para "generar demanda", con aspectos tanto objetivos (como el beneficio que generan), como subjetivo (la percepción de los consumidores). La única marca española que aparecía en la clasificación era Zara.

Una de las novedades de este estudio es la medición del valor de marca de las principales firmas del sector textil y de la confección no estableciendo un ranking sino un posicionamiento con el objeto de identificar cuáles son los principales competidores en relación al valor de marca e identificar sus posibles estrategias.

\section{Objetivo del estudio}

El principal objetivo de este estudio es analizar cómo la marca crea valor para la empresa y medir esta creación en relación con la competencia. Con la presente investigación de trata de averiguar cuál es el posicionamiento actual de las principales firmas de moda.

\section{Breve descripción de los elemen- tos investigados}

Las principales firmas de moda seleccionadas para este estudio son Inditex, H\&M, Gap, Next, Primark, Abercrombie \& Fich, Mango, Cortefield y C\&A. Estas marcas de moda tienen en común que se comercializan en el mercado nacional español pero además tienen presencia internacional (Ver tabla 1). Asimismo han sido objeto de estudios anteriores sobre ranking en el valor de marca.

\section{Tabla 1 - Presencia nacional e internacional de las principales firmas de moda}

\begin{tabular}{|c|c|c|c|c|}
\hline & & $\mathrm{N}^{\circ}$ de Países Presente & $\begin{array}{l}\text { № Tiendas Nacional } \\
\text { (España) }\end{array}$ & No Tiendas Totales \\
\hline 1 & Grupo Inditex & 74 & 1.925 & 4.607 \\
\hline 2 & $H \& M$ & 35 & 114 & 1.988 \\
\hline 3 & Gap & 4 & 1 & 3.100 \\
\hline 4 & Next & 15 & 2 & 510 \\
\hline 5 & Primark & 7 & 19 & 215 \\
\hline 6 & Abercrombie\&Fitch & 49 & 2 & 1.127 \\
\hline 7 & Mango & 92 & 306 & 1.223 \\
\hline 8 & Grupo Cortefiel & 37 & 617 & 1.100 \\
\hline 9 & $C \& A$ & 19 & 121 & 1.467 \\
\hline
\end{tabular}


A continuación se muestra una breve descripción de cada una de las firmas estudiadas:

El grupo Inditex es una conocida marca española a nivel internacional. Este grupo segmentó el mercado, creando para ello 8 marcas comerciales bien diferenciadas (Zara, Massimo Dutti, Pull \& Bear, Berska, Stradivarius, Oysho, Zara Home y Uterqüe). Durante el ejercicio 2010, las ocho cadenas comerciales que integran el Grupo han desarrollado una fuerte expansión internacional.

H\&M fue fundada en 1947 en Västeras, Suecia, bajo el nombre Hennes, de ropa femenina exclusivamente. Más tarde se dedica también a la moda de hombres y niños, cambiando su nombre a Hennes \& Mauritz. Así comenzó una época de gran expansión en varios países. En 2010 ya se encontraba en 35 mercados con 1.988 tiendas y 76.000 empleados (79\% mujeres y $21 \%$ hombres). Su objetivo es tratar de ofrecer calidad en sus productos al mejor precio para el consumidor.

GAP. En 1969, fue fundada por Don Fisher y su esposa Doris. En la actualidad cuentan con unos 135.000 empleados y 3.100 tiendas repartidas en Estados Unidos, Canadá, Reino Unido, Francia, Irlanda y Japón. Esta marca ofrece ropa, accesorios y productos de cuidado personal para hombres, mujeres, niños y bebés bajo las marcas Gap, Old Navy, Banana Republic, Piperlime y Athleta:

- Gap (1969): ropa con estilo clásico, de alta calidad, prendas de vestir casual a precio moderado. Incluye las marcas GapKids (1986), babyGap (1989), GapBody (1998) de ropa interior femenina y Gap Outlet.

- Old Navy (1994): ofrece ropa, zapatos y accesorios, así como una línea de materni- dad, artículos de consumo y productos para el cuidado personal.

- Banana Republic (1983): ropa casual y a medida, zapatos, accesorios y productos para el cuidado personal a un precio superior a Gap. También incluye Banana Republic Factory Stores, con productos similares a precios más bajos.

- Piperlime (2006): tienda online (piperlime.com) con calzado, bolsos, ropa y joyas para mujeres y calzado para hombres y niños; además ofrece consejos y tendencias y asesoramiento.

- Athleta (2008): tienda online (athleta.com) y por catálogo; prendas de vestir y egante y funcional para actividades como correr, esquiar, yoga, jugar al golf, al tenis, o hacer snowboard. Además incluye productos de marcas líderes en ropa de mujer.

La marca compite a nivel local, nacional y mundial con grandes almacenes, tiendas de descuentos, tiendas independientes y empresas online con productos similares. También tienen competencia de cadenas regionales y nacionales de Europa, Japón y Canadá.

Next fue creada en 1982, dedicada a ropa y accesorios de mujer. Desde el principio se ha consolidado como una marca con estilo y buena calidad a un precio asequible. Más tarde amplió su línea de productos a ropa de hombre (1984), de hogar (1985) y de niño (1987). En 1999 creó su página web, y a partir de entonces se podía comprar a través de la tienda, por teléfono o por internet. En 2007 lazó una firma de lujo de moda y artículos para el hogar, y en 2010 creó NX Sports. Actualmente, Next ha sido elegida proveedora oficial para los Juegos Olímpicos de Londres 2012. 
Primark comienza en junio de 1969 con una primera tienda en Mary Street, Dublín, llamada "Penneys". En 1995 adquiere la cadena de moda BHS, comenzando así la gran expansión de la marca. Llegó a España en 2006, cuya primera tienda se abrió en Madrid. En diciembre de 2010 ya tenía 215 tiendas abiertas por varios países: Irlanda (38), España (19), Reino Unido (150), Holanda (2), Portugal (2), Alemania (3) y Bélgica (1).

Abercormbie \& Fitch contaba con 1.127 tiendas en 49 estados, Canadá y Reino Unido El 20 de Marzo de 2009. Tiene como esencia el lujo informal; crean una combinación entre lo clásico y lo sexy. Se ha convertido en una marca idolatrada, dirigida principalmente a un público joven. Además ofrece otros estilos bajo las marcas Abercrombie, Gilly Hicks y Hollister. Abercrombie está dedicada a la ropa de niños, aspirando a parecerse al estilo de Abercrombie\&Fitch. Gilly Hicks ofrece ropa interior femenina, inspirada en el espíritu libre de Sydney, Australia. Hollister Co. es una marca que atrae principalmente a jóvenes y adolescentes, inspirada en el Sur de California.

Mango fue fundada en 1984 en Barcelona por Isak Andic, con 100\% de capital español. En 1992, se abrió la tienda número 99 en España, y comenzó la expansión internacional de la marca con la inauguración de dos tiendas en Portugal, lo que marcó el inicio de la internacionalización ya que en 1997, el volumen de negocio generado en el extranjero superó por primera vez al nacional.

El Grupo Cortefiel, fue fundado en Madrid en 1880. Actualmente está formado por Cortefiel, Springfield, Pedro del Hierro y Women' secret. El Grupo cuenta con oficinas internacionales de compra en España, Hong Kong y la India.
Su plan de expansión geográfico se efectúa a través de tiendas propias y franquicias. La gestión directa se implanta principalmente en Europa, mientras que la franquicia es su primordial vehículo de expansión internacional.

Por último, tenemos la firma C\&A, cuyos inicios se remontan al S. XVII, cuando Clemens y August Brenninkmeijer se dedicaron al comercio de textiles por Europa. En 1841, los hermanos Brenninkmeijer continuaron con la antigua tradición familiar, fundando la empresa C\&A en Sneek (Países Bajos). En 1911 inauguraron su primera tienda en Berlín (Alemania); a partir de ahí comenzó una etapa de expansión con gran éxito, hasta la actualidad.

\section{Metodología}

En la presente investigación se trata de analizar cómo la marca crea valor para la empresa y medir esta creación en relación con la competencia. Por lo que para medir el valor de marca se va a seguir tanto el procedimiento de valoración de la contabilidad de momentos como el método de valoración del coste teórico. Con este método se intenta ser objetivo y no incurrir en más subjetividades que las necesarias para valorar la marca.

Con el fin de descubrir las asociaciones existentes cada marca y las variables utilizadas para medir el valor de marca desde la óptica de las organizaciones detallistas seleccionadas en este estudio, se ha aplicado el análisis factorial de correspondencias (AFC) a los datos obtenidos de los anuarios económico financieros publicados por cada una de las firmas.

Entre las variables consideradas para medir el valor de marca en este análisis se encuentran: el número de países en los que se encuentra la firma con establecimientos físicos, número de tiendas en España, Número de tiendas totales a nivel 
internacional, número de empleados, el EBIT (beneficios antes de intereses e impuestos), inversión en publicidad, y años de operatividad desde su creación.

En el tratamiento de los datos, se ha utilizado el programa informático DYANE, Versión 4, Dise- ño y análisis de encuestas en investigación social y de mercados (Santesmases, 2009).

\section{Resultados}

En la tabla 2 podemos distinguir el posicionamiento del valor de marca de firmas de moda.

Tabla 2 • Análisis factorial de correspondencias Tabla de valores medios

\begin{tabular}{|c|c|c|c|c|c|c|c|c|c|}
\hline & \multicolumn{9}{|l|}{ Marca } \\
\hline & Inditex & H\&M & Gap & Next & Primark & $\begin{array}{l}\text { Abercombie } \\
\text { \&Fich }\end{array}$ & Mango & Cortefiel & C\&A \\
\hline No país & 74 & 35 & 4 & 15 & 7 & 49 & 92 & 37 & 19 \\
\hline $\mathrm{N}$ tiendas & 1.925 & 114 & 1 & 2 & 19 & 2 & 306 & 617 & 121 \\
\hline $\mathrm{N}$ tiendai & 4.607 & 1.988 & 3.100 & 510 & 215 & 1.125 & 1.223 & 1.100 & 1.467 \\
\hline EBIT & 1.089 & 2.112 & 1.104 & 77,74 & 17,85 & 313 & 192,2 & 94,41 & 138,8 \\
\hline No empleados & 92.301 & 76.000 & 135.000 & 20.368 & 27.000 & 94.600 & 8.600 & 10.203 & 43.066 \\
\hline Año opera & 35 & 63 & 41 & 28 & 41 & 118 & 26 & 60 & 169 \\
\hline Publicidad & 0 & 0,04 & 0,05 & 0,03 & 0,03 & 0,04 & 0,04 & 0,03 & 0,04 \\
\hline
\end{tabular}

Inercia total: $\quad 0,750456$

Ji-cuadrado: $\quad 13170,8400$

\begin{tabular}{|c|c|c|c|c|c|c|c|}
\hline EJE 1 & EJE 2 & EJE 3 & EJE 4 & EJE 5 & EJE 6 & EJE 7 & EJE 8 \\
\hline 0,5405 & 0,1105 & 0,0848 & 0,0114 & 0,0033 & 0 & 0 & 0 \\
\hline 72,0203 & 14,7297 & 11,3003 & 1,5131 & 0,4359 & 0,0005 & 0,0001 & 0,0001 \\
\hline$-0,5505$ & $-0,3067$ & 0,3891 & $-0,4172$ & $-0,2275$ & $-0,3983$ & $-0,5528$ & 0,071 \\
\hline 1,7422 & $-0,2424$ & 0,7971 & 1,3114 & 0,5619 & $-0,6683$ & $-0,0097$ & $-0,3087$ \\
\hline 1,8421 & 0,4894 & 0,4381 & $-2,0431$ & $-0,6629$ & 2,0038 & 0,4011 & 0,1456 \\
\hline$-0,5839$ & 1,7594 & 0,4011 & 0,7284 & $-2,9507$ & $-1,4182$ & 3,4199 & 0,2838 \\
\hline$-0,4803$ & 1,8909 & $-1,5668$ & $-0,7228$ & 0,423 & $-0,2454$ & $-0,1849$ & $-6,766$ \\
\hline 1,4425 & 1,471 & $-3,8097$ & $-1,247$ & 0,7458 & $-2,3802$ & $-0,5685$ & 1,6204 \\
\hline 0,323 & $-3,2618$ & $-2,7898$ & 1,3782 & $-2,1617$ & 1,2977 & 0,187 & $-0,4752$ \\
\hline$-0,7607$ & $-0,619$ & $-0,2916$ & $-0,2223$ & 1,9024 & 0,4469 & 1,79 & 0,1739 \\
\hline$-0,6614$ & 1,5317 & $-0,392$ & 1,4292 & 0,1504 & 1,4422 & $-0,7687$ & 0,539 \\
\hline
\end{tabular}


Tabla 2 . (continuación)

Análisis factorial de correspondencias - Representación gráfica de los ejes factoriales

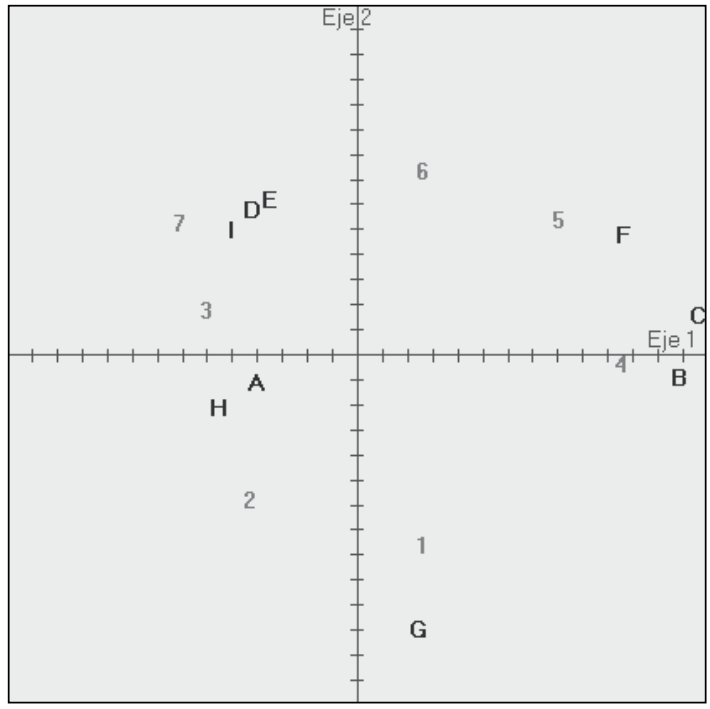

\section{Variables olumna:}

$\begin{array}{cl}\text { Código } & \text { Significado } \\ \text { A } & \text { Inditex } \\ \text { B } & \text { H\&M } \\ \text { C } & \text { Gap } \\ \text { D } & \text { Next } \\ \text { E } & \text { Primark } \\ \text { F } & \text { Abercombie\&Fich } \\ \text { G } & \text { Mango } \\ \text { H } & \text { Cortefiel } \\ \text { I } & \text { C\&A }\end{array}$

\section{Variables fila:}

$\begin{array}{cl}\text { Código } & \text { Significado } \\ 1 & \text { No país }_{2} \\ 2 & \text { N tiendas } \\ 3 & \text { N tiendai } \\ 4 & \text { EBIT } \\ 5 & \text { N }^{o} \text { empleados }\end{array}$

\section{Año opera \\ $7 \quad$ Publicidad}

Como se puede observar en el primer cuadrante se sitúan C\&A, Next y Primark representadas por las letras I, D, y E, respectivamente destacan por su presencia internacional e inversión en publicidad por lo que podemos decir que sus estrategias de internacionalización van acompañadas de estrategias de comunicación.

El valor de la marca de Abercormbie \& Fitch se encuentra reforzado en estrategias de marketing aplicadas en sus empleados como se aprecia en el tercer cuadrante. Esta firma cuida la imagen que comunican sus empleados contratando incluso modelos.

Muy próximos al ejel se encuentran las marcas H\&M y GAP por lo que muestran una fuerte rivalidad en valor de marca. Lo que coincide con los resultados de otros estudios de ranking de 
valor de marca. Ambas marcas destacan por sus fuertes beneficios.

Las marcas más próximas entre sí son Inditex con Cortefiel, representadas por las letras A y H, respectivamente en el tercer cuadrante de abajoizquierda caracterizados por tener mayor presencia nacional. Por lo que su estrategia de diferenciación del valor de marca puede ir enfocada a ser de las marcas más notorias a nivel nacional.

Por último el valor de marca de Mango representado por la letra $G$ se diferencia por su presencia en un gran número de países. Por lo que muestra una clara estrategia de expansión internacional.

\section{Conclusiones y repercusiones}

En la actualidad la marca se convierte en instrumento clave como estrategia de comunicación del valor añadido de las empresas. Una marca fuerte es la forma más eficiente de crear y mantener una valiosa ventaja competitiva. En este sentido, en el sector de la moda cada vez es más importante la apuesta de la marcas en sus estra- tegias de comunicación, sobre todo cuando estas empresas tienen buenas expectativas de crecimiento nacional e internacional.

Siendo conscientes de las limitaciones de este estudio, se deben comentar que al utilizar variables económicas financieras nos permiten recopilar datos objetivos que desde el punto de vista empresarial son una buena herramienta para valorar la marca de cada organización en relación a sus principales competidores.

Mediante la metodología de valoración de marcas seguido en este trabajo aplicado al sector textil se puede detectar las marcas más próximas, es decir, detectar las marcas rivales más fuertes. Incluso permite descubrir cuales son las principales estrategias de diferenciación de cada una de las firmas.

Finalmente, este estudio se podría aplicar en diferentes momentos del tiempo para ver como evolucionan el valor de las marcas ante cambios de estrategia. Y otra posibilidad que ofrece este trabajo es que se podría extender a otros sectores de actividad. 


\section{Bibliografía}

Aaker, D. (1991): Managing Brand Equity, New York, N.Y., The Free Press.

Aaker, D. (1996): Measuring brand equity across products and markets", California Management Review, vol. 38, n 3, Spring, p 102-120.

Arnold, D.A. (1994): Cómo gestionar una marca, Parramón Ediciones S.A, Barcelona.

Blackston, M. (1990): "Price trade-off as a measure of Brand Value", Journal of Advertising Research, vol. 30, n 4, p. 3-7.

Broadbent, S. (1992): "Using Data Better", Admap, January, pp. $48-54$.

Capraro, A.J. and Srivastava, R.K. (1997): "Has the influence of financial performance on reputation measures been overstated?", Corporate Reputation Review, vol. 1, no 1 , pp. 86-93.

Cerviño, J. and Peralba, R. (2003): Leading Brands of Spain, ICEX (Spanish Foreign Trade Institute), Madrid.

Davies, G. y Brito, E. (2004). "Price and quality competition between brands and own brands: A value systems perspective", European Journal of Marketing, Vol 38, (1/2), pgs. 30-55.

Erloz, M. (1995): Aproximación al capital de marca a través del modelo de elección del consumidor: un ensayo con datos agregados. VII Encuentro de profesores de Marketing.

Farquhar, P.H. (1989): "Managing Brand Equity", Marketing Research, vol. 1, n 3, pp. 24-33.

Gómez arias, T. (1995): Utilización de la Integración Jerárquica de la Información en la Medición del Valor de la Marca. VIII Encuentros de profesores de Marketing.

Kapferer, J-N. (1992): Strategic Brand Management - New approaches to creating and evaluating brand equity, London.

Medina, O.; Méndez, J.L. y Rubio, N. (2004). "Price-Quality and market share of consumer goods in Spain: retail brands and manufacturer brands", International Review of Retail, Distribution and Consumer Research, Vol. 14, n 2, pgs. 199-222.
Murphy, J.(1991): Brand Valuation, Business Books Limited, London.

Oubiña, J.; Rubio, N. y Yagüe, M.J. (2006). "Strategic management of store brands: an analysis from the manufacturer's perspective", International Journal of Retail and Distribution Management, Vol. 34, n ${ }^{\circ}$ 10, pgs. 742-760.

Park, CH.S. y Srinivasan, V. (1994): "A survey-based method for measuring and understanding brand equity and its extendibility". Journal of Marketing Research, vol. 31, mayo, pgs. $271-288$

Rangaswamy, A., Burke, R.R. y Oliva, T.A. (1993): "Brand equity and the extendibiliy of brand names". International Journal of Research in Marketing, vol. 10, núm. 1, marzo, pgs. 61-75.

Rubinstein, H. (1996): “'Brand First' Management”, Journal of Marketing Management, Vol. 2, pp. 269-280.

Simon, C.J. and Sullivan, M. W. (1993): "The measurement and determinants of brand equity: A financial approach", Marketing Science, vol. 12, n 1, Winter, pp. 28-52.

Srivastava, R.K., Shervani, T.A. and Fahey, L. (1998): "Market-based Assets and Shareholder Value: A Framework for Analysis", Journal of Marketing, Vol. 62, January, pp. 2-18.

Woodall (2003), T. (2003): "Conceptualising 'Value for the customer': An attributional, structural and dispositional analysis". Academy of Marketing Science Review, vol. 2003, n 12 , pgs. 1-42.

Yoo, B., Donthu, N. y Lee, S. (2000): "An examination of selected marketing mix elements and brand equity". Journal of Academy of Marketing Science, vol. 28, núm. 2, primavera, pgs. 195-211.

Yustas, Y. y Requena, M. (2010). "Marcas de la distribución. Denominación y definición para una comunicación más precisa". ADResearch ESIC. Revista Internacional de Investigación en Comunicación. Pgs. 76-95. 